\title{
RNA Interference and Nonviral Targeted Gene Therapy of Experimental Brain Cancer
}

\author{
Ruben J. Boado \\ ArmaGen Technologies, Inc., Santa Monica, California 90401; and Department of Medicine, UCLA, Los Angeles, \\ California 90024
}

\begin{abstract}
Summary: The human epidermal growth factor receptor (EGFR) plays an oncogenic role in solid cancer, including brain primary and metastatic cancers. Transvascular nonviral gene therapy in combination with EGFR-RNA interference (RNAi) represents a new therapeutic approach to silencing oncogenic genes in solid cancers. This is achieved with pegylated immunoliposomes (PIL) carrying short hairpin RNA expression plasmids driven by the U6 RNA polymerase promoter and directed to target EGFR expression by RNAi. The PIL is comprised of a mixture of known lipids containing polyethyleneglycol (PEG), which stabilizes the PIL structure in vivo in circulation. The tissue target specificity of PILs is given by conjugation of $\sim 1 \%$ of the PEG residues to monoclonal antibodies (mAbs) that bind to specific endogenous receptors (i.e., insulin and transferrin receptors) located in the brain vascular endothelium,
\end{abstract}

which forms the blood brain barrier (BBB), and brain cellular membranes, respectively. These mAbs are known to induce 1) receptor-mediated transcytosis of the PIL complex through the $\mathrm{BBB}$ and 2) transport to the brain cell nuclear compartment. Treatment of an experimental human brain tumor model in scid mice is possible with weekly intravenous RNAi gene therapy causing reduced tumor expression of EGFR and $88 \%$ increase in survival time of these mice with advanced intracranial brain cancer. The availability of additional RNAi tumor targets may improve the therapeutic efficacy of this new anticancer drug. The accessibility to chimeric and/or humanized mAbs directed to human BBB and brain cell specific-receptors may accelerate the application of this technology to the treatment of human tumors. Key Words: Blood-brain barrier, brain tumor, gene therapy, liposomes, RNA interference, short hairpin RNA.

\section{INTRODUCTION}

The human glioblastoma multiform (GBM) represents the most malignant form of human astrocytomas, and approximately 15,000 new cases are reported per year in the U.S. alone. ${ }^{1}$ In addition, the incidence of metastatic brain tumors approximates 150,000 cases per year in the U.S., and these include lung, breast, and melanoma metastasis, respectively. ${ }^{1}$ The survival rate for patients with brain primary and metastatic cancers is poor after conventional therapy, including surgery, radiation therapy, and/or chemotherapy. ${ }^{2-7}$ Therefore, development of new therapeutics is critical to improve the life expectancy of these patients.

The human epidermal growth factor receptor (EGFR) represents a potential target for the development of new therapeutics for the treatment of cancers in general. ${ }^{8,9}$ The EFGR drives the proliferation of $90 \%$ of GBM. ${ }^{9,10}$

Address correspondence and reprint requests to Dr. Ruben J. Boado, Department of Medicine, UCLA Warren Hall 13-164, 900 Veteran Avenue, Los Angeles, CA 90024. E-mail: rboado@mednet.ucla.edu.
The EGFR also plays an oncogenic role in approximately $70 \%$ of the peripheral solid cancers that metastasize to the brain. ${ }^{11}$ Therefore, if therapeutics to target the EGFR-responsive brain tumor were developed, this may result in the potential treatment of approximately 100,000 cases per year just in the U.S. Amplification of the EGFR gene has been described in more the $40 \%$ of $\mathrm{GBM},{ }^{12,13}$ and there is strong evidence for association between overexpression and gene amplification of EGFR in brain cancers. ${ }^{14,15}$ In addition to the amplification of the wild-type EGFR gene in human brain tumors, there are mutant forms of EGFR that may be constitutively overactive, and the most common form of mutant EGFR has an in frame deletion of exons 2-7, and it is designated EGFR variant III (vIII). ${ }^{9}$ The EGFR vIII protein is poorly responsive to its ligands, i.e., EGF and TGF. However, this mutant maintains the tyrosine kinase domain of the EGFR in continuously hyperactivity resulting in over proliferation of the cancer cells. ${ }^{9}$ Therefore, overexpression of the EGFR VIII protein and EGFR gene amplification have been associated with poor survival. ${ }^{16}$ Based on the biochemical characteristics of the EGFR 
and oncogenic role, potential therapeutics for brain cancers should be able to target both wild-type and mutant forms of the EGFR.

The development of a new therapeutic for either primary or metastatic cancer of the brain (i.e., small molecules, monoclonal antibodies, or gene medicines) will not succeed in clinical trials if the drug does not cross the brain microvasculature, which constitutes the bloodbrain barrier (BBB) in vivo. ${ }^{17}$ The $\mathrm{BBB}$ is formed by blood vessels that originate from normal brain and which perfuse the primary or metastatic cancer in brain. Even though is it generally accepted that there is an increased permeability of the BBB in high grade gliomas (i.e., GBM), in the early and intermediate stages of brain cancer when therapeutic intervention is desirable, the capillaries perfusing the brain cancer have restrictive permeability properties similar to capillaries in normal brain. ${ }^{18}$ The brain microvascular barrier is only permeable to lipophilic molecules of less than $500 \mathrm{Da}$, and the majority of potential drug candidates to target the EGFR do not cross the BBB, resulting in poor therapeutic efficacy in brain cancers. For example, kinase small-molecule inhibitors, such as PTK787 or Gleevec (Novartis Pharmaceuticals, East Hanover, NJ), do not cross the BBB. ${ }^{19}$ The efficacy of gefitinib (Iressa, AstraZeneca, Newark, DE) for brain cancers remains to be determined in clinical trials. However, this compound was recently shown to be effective primarily in particular human somatic mutations of EGFR, which represent a small percentage of the human population. ${ }^{20,21}$ Therefore, the potential clinical application of Iressa for the treatment of human primary tumors of the brain is reduced to this small percentage of patients carrying these single point mutations. ${ }^{20,21}$ In addition, even if this drug is active in the treatment brain primary tumors overexpressing the EGFR in this particular population, preclinical data show that Iressa will not be effective in primary or metastatic brain cancers expressing mutants of the EGFR such as the EGFR vIII. ${ }^{22}$ Monoclonal antibodies (mAbs) that block the EGFR may be specific for either the wild-type EGFR or the mutant EGFR, i.e., vIII. ${ }^{23,24}$ These mAbs may be effective in inhibiting their specific oncogenic stimulation pathway in peripheral cancers. Nevertheless, the potential clinical application of these mAbs for brain cancers is diminished because mAbs do not cross the BBB. ${ }^{17}$ The problem presented by the BBB in the development of brain cancer therapeutics is illustrated in the case of Herceptin (Genentech, Vacaville, CA), a humanized monoclonal antibody to the HER2 receptor, which is a member of the EGFR gene family. Although Herceptin inhibits growth of HER2-positive cancer in the breast, this therapeutic is not effective against breast cancer that has metastasized to the brain. ${ }^{25}$

\section{POTENTIAL TARGET FOR BRAIN CANCER GENE THERAPY}

Gene therapy of brain cancer offers the promise of knocking down the expression of oncogenic genes such as EGFR. However, gene therapy is limited by the delivery problem, which is particularly difficult in brain owing to the presence of the BBB. ${ }^{17}$ To circumvent the $\mathrm{BBB}$, attempts have been made to deliver therapeutics to brain cancer by craniotomy. However, this approach is not effective because there is limited diffusion of the therapeutic gene within the tumor from the transcranial injection site. ${ }^{26}$ Therapeutics can be delivered to all cells in brain cancer via the transvascular route across the BBB. ${ }^{17,27}$ The transvascular delivery of nonviral genes to brain is possible with a gene transfer technology that uses pegylated immunoliposomes (PILs). ${ }^{27,28}$

The application of the PIL nonviral gene transfer technology enabled a significant increase in survival time of mice with intracranial human brain cancer with weekly intravenous injections of antisense gene therapy directed at the human EGFR. ${ }^{29}$ In this experimental therapy approach, the antisense construct (designated clone 882) is comprised of an eukaryotic expression plasmid encoding a 700-nucleotide RNA that is antisense to nucleotides 2317-3006 of the human EGFR encompassing the kinase domain of this receptor. ${ }^{30}$ The antisense clone 882 was encapsulated in PILs that were doubly targeted to brain cancer in vivo with two mAbs of different receptor specificity. ${ }^{29}$ One $\mathrm{mAb}$, the rat $8 \mathrm{D} 3 \mathrm{mAb}$ to the mouse transferrin receptor (TfR) (FIG. 1), enabled transport of the PIL across the mouse BBB forming the microvasculature of the intracranial cancer. A second mAb targeted the human insulin receptor (HIR) that was expressed on the human brain cancer plasma membrane (FIG. 1). To augment the potency of the clone 882 expression plasmid, this vector contained the latent origin of plasmid replication (oriP) and Epstein-Barr nuclear antigen (EBNA)-1 elements, ${ }^{30}$ which allow for a single round of replication of the expression plasmid with each division of the cancer cell. ${ }^{31}$ The inclusion of the oriP/EBNA-1 elements within the expression plasmid enables a 10-fold increase in the level of gene expression in human U87 glioma cells. ${ }^{32}$ However, the EBNA-1 gene encodes a tumorigenic transacting factor, ${ }^{33}$ and this formulation may not be desirable in human gene therapy. It is possible that the EBNA-1 element would not be required if a more potent form of antisense gene therapy was developed.

RNA interference (RNAi) is a new form of antisense gene regulation wherein short RNA duplexes of defined sequence results in gene silencing of the targeted mRNA. ${ }^{34}$ RNAi is perhaps the most potent mechanism of gene downregulation. RNAi was named "Breakthrough of the Year" by Science in 2002 as important mechanism of gene regulation. ${ }^{35}$ 


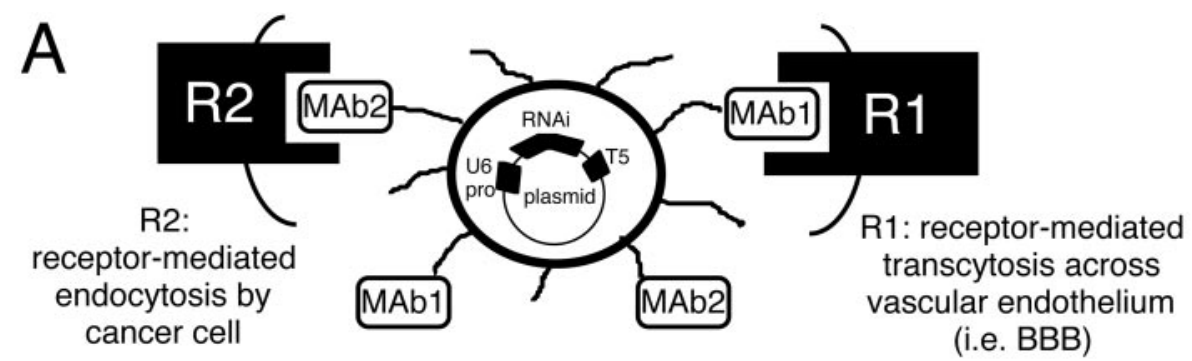

\begin{tabular}{|c|c|c|}
\hline \multicolumn{3}{|c|}{ Engineering of PILs } \\
\hline Targeting Mab & Target Receptor & $\begin{array}{l}\text { Experimental Model and } \\
\text { Target Tissues }\end{array}$ \\
\hline OX26 & Rat TfR & $\begin{array}{l}\text { a) Rat C6 glioma in cultures. } \\
\text { b) Rat C6-790 glioma in cultures. } \\
\text { c) In vivo transport via rat BBB and rat brain cell } \\
\text { (neuron and glial) gene delivery. }\end{array}$ \\
\hline 8D3 & Mouse TfR & $\begin{array}{l}\text { In vivo transport via mouse BBB and mouse brain } \\
\text { cell (neuron and glial) gene delivery. }\end{array}$ \\
\hline 8314 & Human IR & $\begin{array}{l}\text { a) Human U87 glioma cultures. } \\
\text { b) In vivo transport via human BBB and human brain } \\
\text { cell (neuron and glial) gene delivery. }\end{array}$ \\
\hline $8 D 3+8314$ & $\begin{array}{c}\text { Mouse TfR + } \\
\text { Human IR }\end{array}$ & $\begin{array}{l}\text { Experimental human brain tumors in scid mice. In } \\
\text { vivo transport via mouse BBB and human glioma } \\
\text { gene delivery. }\end{array}$ \\
\hline
\end{tabular}

FIG. 1. Engineering of PIL. Top: PIL with supercoiled plasmid DNA encapsulated in the interior of the liposome. The gene encoding the shRNA is under the influence of the U6 promoter and the coding region terminates with the T5 terminator sequence for RNA polymerase III. The surface of the liposome is conjugated with several thousand strands of 2000 Da PEG to stabilize the liposome in the blood. ${ }^{44}$ The tips of $1-2 \%$ of the PEG strands are conjugated with a targeting ligand such as a receptor (R)-specific mAb, which triggers transport of the PIL across biological barriers in vivo. Bottom: PILs can be engineered to target cells in tissue culture and in vivo experimental models in different species. For example, PILs constructed with both 8D3 mouse TfR mAb and the 8314 human insulin receptor (IR) mAb are transported through the BBB via receptor mediated transcytosis, and it will target human glioma cells in an experimental mouse model of human brain tumor via endocytosis. ${ }^{29,41-46}$

RNAi has been demonstrated in cell culture by lipofection with RNA duplexes. However, the delivery of short RNA fragments to cells in vivo in mammals is problematic owing to the rapid degradation of the RNA. Short hairpin RNA (shRNA) mimics the structure of the RNAi duplex, and shRNAs can be produced in cells after the delivery of expression plasmids encoding the shRNA. This shRNA is processed in the cell by an enzyme called dicer to form an RNA duplex with a 3'-overhang, and this short RNA duplex mediates RNAi or posttranscriptional gene silencing. ${ }^{36,37}$ RNAi activity has been shown in cell culture by transfecting cells with plasmids producing shRNAs, using gene delivery systems comprised of either cationic polyplexes or retroviral vectors. ${ }^{36-40}$ However, cationic DNA polyplexes (i.e., lipofection) or retroviral vectors do not cross the $\mathrm{BBB}$ and do not allow for gene delivery to the brain. ${ }^{17} \mathrm{Al}$ though RNAi-based gene therapy offers promise for the treatment of cancer, the limiting factor is delivery. Recent studies demonstrated that it is possible to engineer delivery systems for shRNA expression vectors with therapeutic efficacy directed at the human EGFR in an experimental human brain tumor model in mice. ${ }^{41}$

\section{GENE DELIVERY OF ShRNA EXPRESSION VECTORS}

Short hairpin RNA mimics the structure of the RNAi duplex, and shRNAs can be produced in cells following the delivery of expression plasmids encoding the shRNA. However, this reconfigures the formulation of the potential RNA drug into a DNA gene medicine. Recently, a new form of nonviral gene transfer has been developed that enables efficient expression of plasmid DNA in target organs following an intravenous injection of the gene. ${ }^{42,43}$ The plasmid DNA is encapsulated in the interior of an $85 \mathrm{~nm}$ liposome (FIG. 1), which protects the DNA from the ubiquitous endonucleases in vivo. The surface of the liposome is conjugated with several thousand strands of 2000 Da polyethyleneglycol (PEG), and the tips of $1-2 \%$ of the PEG strands are tethered with a 
targeting ligand acting as a molecular Trojan horse. The structure of the PIL carrying the internalized plasmid DNA is shown in Figure 1. The PILs may be engineering with different peptidomimetic mAbs that bind to different endogenous receptors to induce receptor-mediated transcytosis through the $\mathrm{BBB}$, and transport via endocytosis to the nuclear compartment in brain cells including cancer cells. ${ }^{42-44}$ For example, the 8314 murine mAb to the HIR and the OX26 murine mAb to the rat TfR are used to target human and rat tissues, respectively. The OX26 TfRmAb is active only in rats, and the 83-14 HIRmAb is active only in humans or Old World primates such as the rhesus monkey (FIG. 1). ${ }^{32,42-46}$

The targeting $\mathrm{mAb}$ delivers the PIL carrying the gene across the biological barriers in vivo. ${ }^{42-47}$ In the case of targeting brain cancer, the PIL must traverse both the $\mathrm{BBB}$ in vivo, and the tumor cell plasma membrane (TCM) behind the BBB. Owing to high expression of the TfR or IR on both the BBB and TCM barriers, the targeting $\mathrm{mAb}$ enables the sequential receptor-mediated transcytosis of the PIL across the BBB followed by the receptor-mediated endocytosis of the PIL into the brain tumor cell in a brain tumor model. ${ }^{44}$ PILs have also been successfully constructed to target human tumor cells in a scid mouse model wherein dual targeting mAbs were directed to the mouse TfR and human IR. ${ }^{41}$

\section{IN VIVO SILENCING OF GENE EXPRESSION IN A BRAIN TUMOR MODEL: LUCIFERASE TARGET}

The limiting problem in the development of RNAibased therapeutics for cancer and other diseases is the design and construction of shRNA expression plasmid and the delivery of these constructs to the target organ in vivo. Proof of this concept was recently demonstrated with the production of PIL encapsulated antiluciferase shRNA expression plasmids and an intracranial brain cancer model of rat glioma cells permanently transfected with the luciferase gene. ${ }^{44}$

The construction of antiluciferase shRNA expression plasmids is summarized in Figure 2. Different shRNA expression plasmids, designated clones 952 and 954, were prepared that hybridize to overlapping sequences of the luciferase mRNA sequence shown in Figure 2A. This area of the luciferase mRNA was selected because previous work demonstrated that it was possible to produce silencing of the luciferase gene with shRNA. ${ }^{36}$ Complementary oligodeoxynucleotides (ODNs) were synthesized to produce the duplexes corresponding to either the clone 952 or clone 954 shRNA. The structures of the shRNA encoded by clone 952 and 954 are shown in Figure 2, B and C, respectively. The clone 952 shRNA has the antisense strand on top with a 25 -mer stem and an 8-nucleotide loop, whereas the clone 954 shRNA con- tains the antisense strand on the bottom with a 20-mer stem and a 7-nucleotide loop (FIG. 2, B and C). The sequence complementary to the forward ODN contains 4-nucleotide overhangs to the EcoRI and ApaI restriction sites at $5^{\prime}$ - and $3^{\prime}$-end, respectively (FIG. 2D), to direct subcloning into the cohesive ends of the expression vector driven by the U6 promoter shown in Figure 1A. ${ }^{37}$ The T5 terminator sequence for RNA polymerase III was introduced in the ODNs (FIG. 2D).

The biological activity of antiluciferase shRNA expression plasmids was investigated in human U87 glioma cells in tissue culture. Cells were cotransfected with a luciferase expression vector clone 790 and clones 952 or 954 by lipofection. Clone 790 is a pCEP4-derived expression plasmid driven by the simian virus 40 (SV40) promoter with a cis-stabilizing element in the $3^{\prime}$-untranslated region (UTR) of the luciferase mRNA. ${ }^{47}$ In the cells transfected with clone 954 there was an $\sim 60 \%$ inhibition in luciferase gene expression at 2 days of incubation, and this effect was lost at day 4 (FIG. 2E, left). Conversely, in the cells transfected with clone 952 , the luciferase activity was suppressed $91 \%$ and $87 \%$ at 2 and 4 days of incubation, respectively (FIG. 2E, left). These studies with lipofection indicated both clones 952 and 954 were active in producing antiluciferase shRNAs, but clone 952 produced maximum silencing of the luciferase gene.

Before animal studies, the ability of the PIL gene delivery system to effectively produce RNAi in cultured cells was validated with the same shRNA expression plasmids (FIG. 2E, right). Clone 952 or 954 shRNA expression plasmids were encapsulated in antibody-targeted PILs as described in Figure 1 using the 8314 HIR mAb to target human U87 glioma cells. ${ }^{30}$ For this experimental design, cells were transfected with the luciferase expression vector clone 790 by lipofection $4 \mathrm{~h}$ before the experiment. Thereafter, cells were incubated with clone 952 or 954 plasmid DNA encapsulated in PILs targeted with the $8314 \mathrm{mAb}$ to the HIR. Clone 954 encapsulated in the HIR mAb-PIL reduced luciferase gene expression 69 and $26 \%$ at 2 and 4 days of incubation, respectively (FIG. 2E, right). In contrast, clone 952 encapsulated in HIR mAb-PIL suppressed luciferase gene expression 92 and $83 \%$, respectively, at 2 and 4 days of incubation (FIG. 2E, right). Data suggest that the PIL gene delivery system could enable RNAi via shRNA production in cultured cells, and that the activity of these complexes is just as high in culture whether the PILs or lipofection was used as the transfection agent (FIG. 2E, right). As seen above with lipofection, clone 952 produced a higher inhibitory effect on the target gene than those observed with clone 954; therefore, clone 952 was used for further studies in animals. 
A 954 luciferase mRNA

5 ' - $\frac{954}{\text { AUACAAAGGAUAUCAGGUGGCCCUCGCUGAAUUGGA }}-3$ '

B

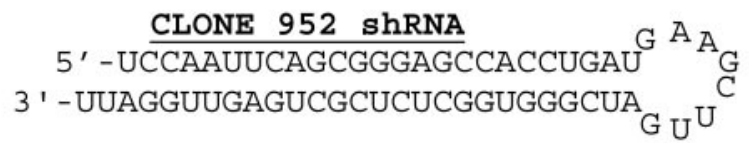

C

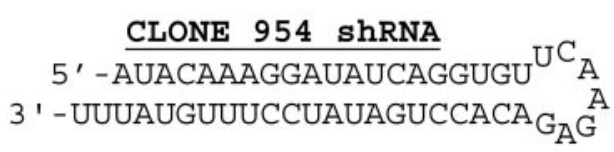

D 5 ' - TCCAATTCAGCGGGAGCCACCTGATGAAGCTTGATCGGGTGGCTCTCGCTGAGTTGGATTTTTTTT- 3 ' 3 ' - CCGGAGGTTAAGTCGCCCTCGGTGGACTACTTCGAACTAGCCCACCGAGAGCGACTCAACCTAAAAAAAATTAA-5 '

E

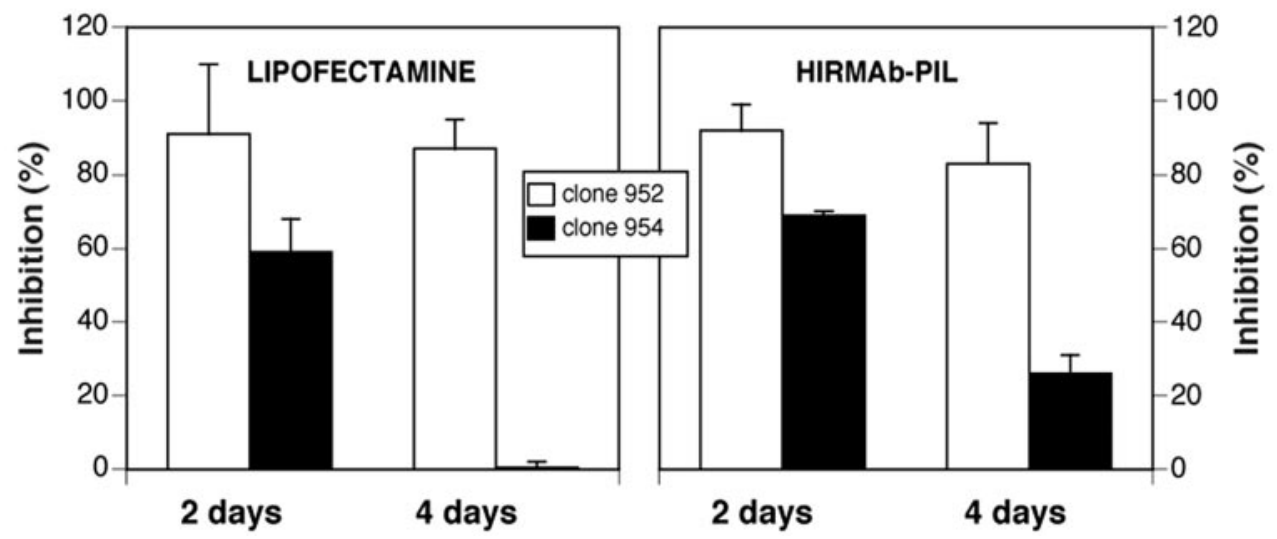

FIG. 2. A: Target sequence of the luciferase mRNA. The sequence targeted by the clone 952-derived shRNA is underlined, and the sequence targeted by the clone 954-derived shRNA is overlined. B and C: Sequences and secondary structure of shRNAs encoded by clone 952 and clone 954, respectively. D: For the construction of clone 952 shRNA expression vectors, complementary ODNs are synthesized and annealed. The sequence complementary to the forward ODN contains 4-nucleotide overhangs to the EcoRI and Apal restriction sites at $5^{\prime}$ - and $3^{\prime}$-end, respectively, to direct subcloning into the cohesive ends of the expression vector driven by the U6 promoter shown in Figure 1A. ${ }^{37}$ The T5 terminator sequence for RNA polymerase III is introduced in the sequence of the ODNs. E, left: Percent inhibition in luciferase activity at 2 and 4 days caused by cotransfection at zero time of the U87 cells with clone 790 , a pCEP4-derived luciferase expression plasmid, ${ }^{48}$ and shRNA clones 952 or 954 plasmid DNA, respectively. There is no inhibition of luciferase expression in the cells transfected with clone 954 at 4 days. E, right: Percent inhibition in luciferase activity at 2 and 4 days caused by exposure of the U87 cells to PIL encapsulated clone 952 and 954, respectively. Cells were exposed to lipofectamine and clone 790 plasmid DNA for $4 \mathrm{~h}$, washed, and the HIRmAb-PILs carrying either clone 952 or clone 954 were added, and the cells were incubated for 2 or 4 days before measurement of luciferase activity. All data are mean \pm SEM ( $n=3$ dishes per point). Reproduced with permission from Zhang et al. In vivo knockdown of gene expression in brain cancer with intravenous RNAi in adult rats. $J$ Gene Med 5:1039-1045. Copyright (c) 2003, John Wiley \& Sons, Ltd. All rights reserved. ${ }^{44}$

The efficacy of clone 952 in silencing the luciferase gene was investigated in vivo in a rat brain tumor model (FIG. 3). C6 rat glioma cells permanently transfected with the luciferase expression plasmid clone $790,{ }^{47}$ and designated C6-790 cells, were injected into caudate-putamen nucleus of Fischer CD344 adult rats. These animals developed large intracranial brain cancers, and the size of the cancer at 14 days after implantation is shown in Figure 3A. The rats were intravenously injected with either saline or $10 \mu \mathrm{g} / \mathrm{rat}$ of clone 952 plasmid DNA encapsulated in PILs at 10 days after tumor implantation, when the tumors occupied about $25 \%$ of the cranial volume. The PILs were constructed with the OX26 TfR $\mathrm{mAb}$ to target transcytosis through the $\mathrm{BBB}$ and gene delivery to brain tumor cells (FIG. 1). ${ }^{44}$ Animals were sacrificed at 12 and 14 days after tumor implantation, which represents 2 and 4 days after a single intravenous injection of the clone 952 plasmid DNA encapsulated in the TfR mAb-PIL. Luciferase gene expression was inhibited $68 \%$ on day 2 after intravenous administration of the shRNA plasmid encapsulated in the PIL (FIG. 3B). As expected, the luciferase gene expression in the contralateral brain was negligible when compared with the levels of luciferase gene expression seen in the tumor (FIG. 3B). Luciferase gene expression was also inhibited at 4 days after the single intravenous injection of the clone 952-PIL. ${ }^{44}$ In contrast to luciferase, the RNAi therapy caused no change in tumor levels of GTP used as control gene (FIG. 3C). This enzyme is expressed in many cancers ${ }^{48}$ including C6 glioma cells. ${ }^{49}$ A knock- 


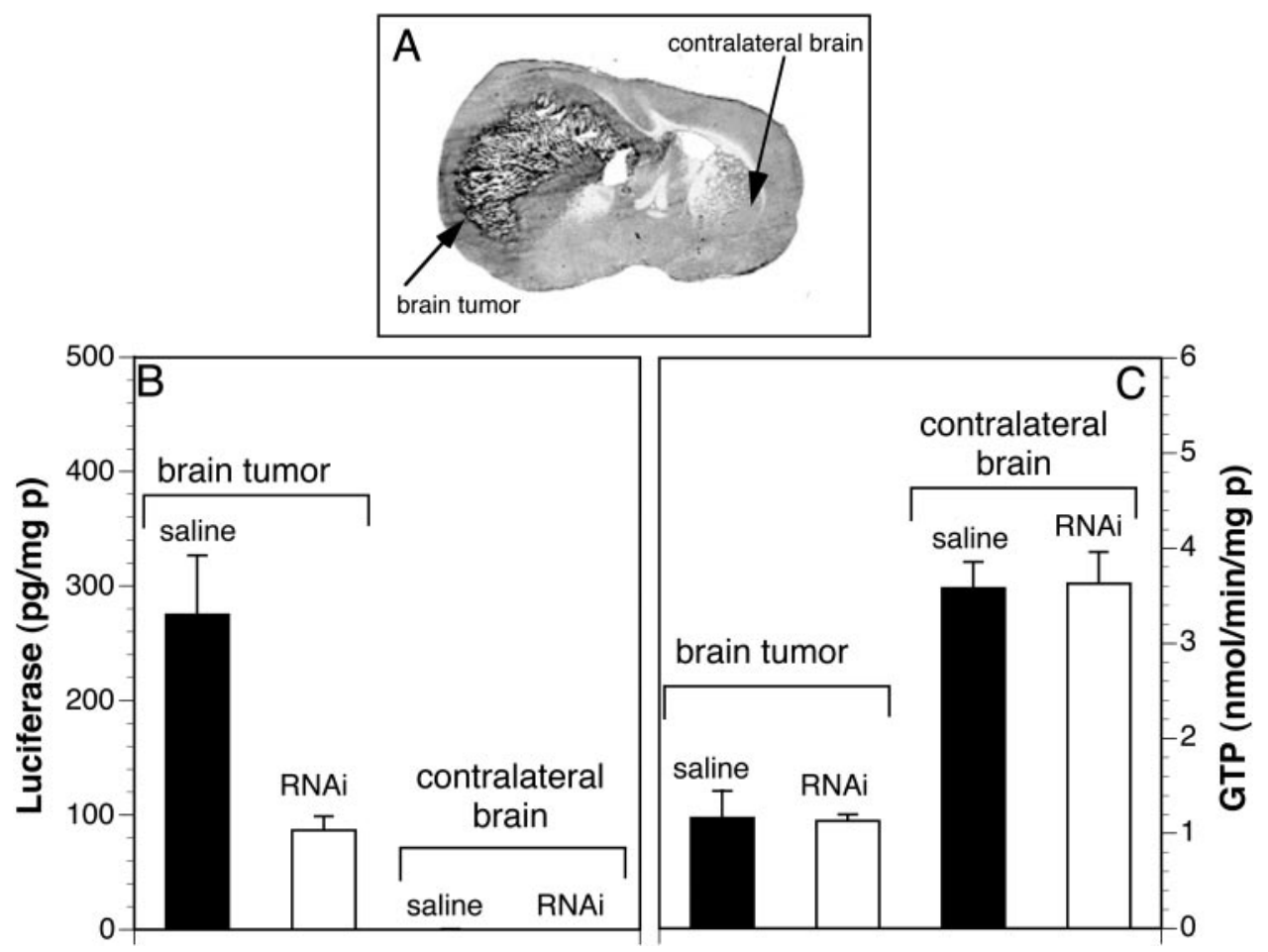

FIG. 3. A: Coronal section of autopsy rat brain at 14 days after implantation of C6-790 rat glioma cells in the caudate-putamen of adult Fischer CD344 rats (180-200 g). The C6 cells were permanently transfected with clone 790 plasmid DNA, and produce high levels of luciferase when grown as brain tumors in vivo. ${ }^{44} \mathrm{~B}$ : Luciferase activity in brain tumor and contralateral brain of controls (saline injected) and shRNA-952 TfR mAb-PIL treated rats. At 10 days after C6-790 tumor implantation, the rats were intravenously injected with either saline or $10 \mu \mathrm{g} / \mathrm{rat}$ of clone 952 plasmid DNA encapsulated in PILs conjugated with the OX26 TfR mAb to target delivery through the BBB and gene delivery to brain tumor cells. Animals were sacrificed 2 days later and luciferase activity quantified. C: GTP activity in brain cancer and contralateral brain showing that PIL treatment does not alter the expression pattern of this maker. Data are mean \pm SEM ( $n=4-5$ rats per point). Reproduced with permission ${ }^{44}$ (see FIG. 2 legend).

down of the tumor luciferase gene expression of more than $90 \%$ was also reported when the injection of the shRNA clone 952-PIL was performed at day 5 after tumor implantation in lieu of day $10 .^{44}$ This level of gene inhibition in vivo was comparable with that observed in cell culture (FIG. 2). ${ }^{44}$

The mAb targeted PIL gene delivery system is just as active as lipofectamine in cell culture (FIG. 2), and this formulation is stable in vivo and allows for global gene delivery to the brain after an intravenous injection. ${ }^{42,45}$ The combination of the PIL gene delivery system and shRNA expression plasmids allows for a $90 \%$ knockdown of brain cancer-specific gene expression. ${ }^{44}$ This effect persists for at least 5 days after a single intravenous injection of a low dose of plasmid DNA, i.e., 10 $\mu \mathrm{g} / \mathrm{rat} .{ }^{44}$ This dose of plasmid DNA is estimated to deliver $\sim 5-10$ plasmid DNA molecules per brain cell in the rodent, ${ }^{50}$ which indicates the PIL gene delivery system to brain has a high efficiency of in vivo transfection.

In vivo RNAi is enabled with this new form of gene delivery system that encapsulates expression plasmids in PILs, which are targeted to distant sites based on the specificity of a receptor-specific monoclonal antibody (FIGS. 1 and 3).

\section{RNAi GENE THERAPY OF BRAIN TUMORS: EGFR TARGET}

A logical extension to this work was to determine if this new technology comprised of shRNA expression vectors and PILs was applicable to the treatment of brain cancer by silencing of genes participating in the oncogenic growth of these tumors, i.e., the EGFR. ${ }^{41}$ The discovery of RNAi-active target sequences within the human EGFR transcript required several iterations wherein successive generation of shRNA expression plasmids were developed (FIG. 4). These findings were consistent with the suggestion of McManus and Sharp, ${ }^{34,51}$ that approximately one of five target sequences yield therapeutic effects in RNAi. A total of six anti-EGFR shRNA encoding expression plasmids were produced and designated clones 962-964 and 966-968 (FIG. 4). Three of these constructs targeted the kinase domain of EGFR (i.e., clones 966-967). Clone 962 was directed to the beginning of the open reading frame (ORF) and clone 964 is complementary to an area near the end of the ORF (FIG. 4). Clone 963 targeted the 5 '-flanking region of the EGFR kinase domain (FIG. 4). The sequence of the antisense strand of each of the six 

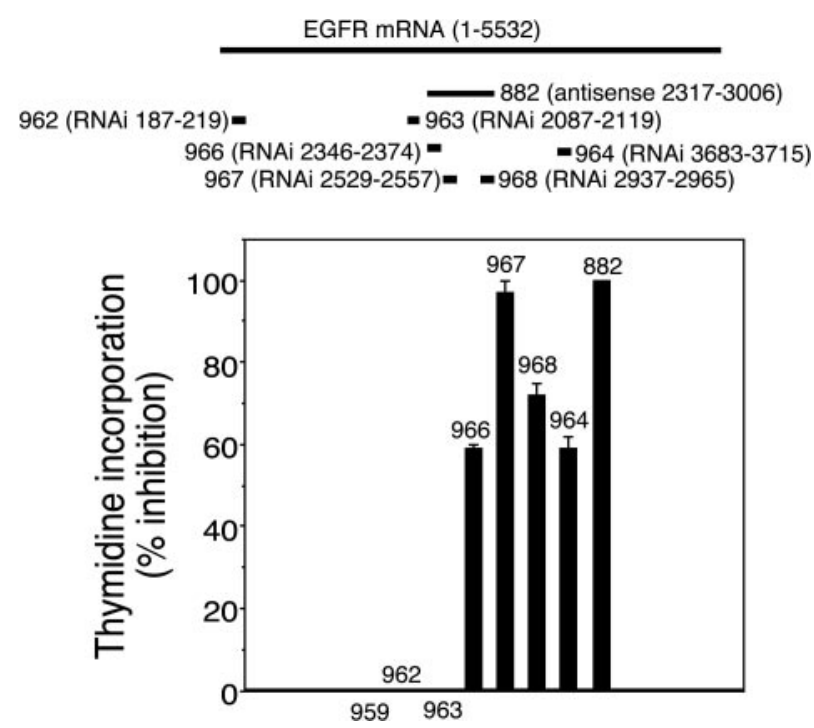

FIG. 4. Screening of shRNA constructs directed to the EGFR. Top: A series of shRNA constructs directed to the EGFR mRNA were prepared. Both nucleotide number and the relative position in the target EGFR mRNA are indicated in the figure. The U6 shRNA expression vectors were prepared as described in Figure 2 for luciferase shRNA plasmids. Bottom: The RNAi efficacy of anti-EGFR constructs was investigated in human U87 glioma cells incubated with $\left[{ }^{3} \mathrm{H}\right]$-thymidine for a 48 -h period that follows lipofection with the plasmid DNA of interest. The shRNA clone 967 and the control antisense-RNA plasmid clone 882 produced maximum inhibitory effect in the incorporation of thymidine in human U87 cells. Clone 882 is a 700-nt antisense RNA complementary to nt 2317-3006 of the human EGFR driven by the SV40 promoter and containing the EBNA-1/oriP elements. ${ }^{30}$ Data are mean \pm SEM $\left(n=3\right.$ dishes). Reproduced with permission ${ }^{41}$ (see Table 1 legend).

shRNAs matches $100 \%$ with the target sequence of the human EGFR (accession number X00588), and they were all directed to the ORF of the EGFR (FIG. 4). The shRNA constructs were designed as previously described $^{36,41}$ and encompass intentional nucleotide mismatches (i.e., G-U) in the sense strand (FIG. 5B) to reduce the hybridization of DNA hairpins during cloning. Because the antisense strand remains unaltered, these substitutions do not interfere with the RNAi effect. ${ }^{52}$ The shRNA expression cassette is engineered with two overlapping ODNs as described in Figure 2D for the luciferase constructs. The EGFR knockdown potency of these six shRNA encoding expression plasmids was compared to the EGFR knockdown effect of clone 882 , known to reduce the expression of this receptor in human glioma cells. ${ }^{29,30}$ Clone 882 has been described above and encodes for a 700-nt antisense RNA complementary to nt 2317-3006 of the human EGFR ${ }^{30}$ (FIG. 4).

The RNAi effect on the human EGFR was investigated by measuring the rate of $\left[{ }^{3} \mathrm{H}\right]$-thymidine incorporation into human U87 glioma cells in tissue culture (FIG. 4) because the EGFR mediates thymidine incorporation into EGFR-dependent cells. ${ }^{53} \mathrm{~A}$ wide range in the response on the RNAi effect on $48-\mathrm{h}\left[{ }^{3} \mathrm{H}\right]$-thymidine incorporation was seen (FIG. 4). For example, no significant inhibition was observed with shRNA constructs 962 and 963 targeting nucleotides 187-219 and 20872119 of the EGFR mRNA, respectively, and with the negative control clone 959 coding for an empty U6 expression vector (FIGS. 1 and 4). On the contrary, clone 967 (nt 2529-2557) was the most potent clone causing an RNA interference of EGFR that was similar to the positive control antisense RNA clone 882. In addition, other shRNA constructs, i.e., 966 and 968 targeting nt 23462374 and 2937-2965, had intermediate effect in the knockdown of EGFR function (FIG. 4).

The thymidine incorporation assays were confirmed by Western blotting, which showed that clones 967 and 882 knockdown the EGFR (FIG. 6). In contrast, clone 962, which has no effect on thymidine incorporation into U87 cells (FIG. 4), also has no effect on the expression of the immunoreactive EGFR (FIG. 6). Similarly, clone 952, which produces an antiluciferase shRNA (FIG. 2B), ${ }^{44}$ has no effect on the EGFR either (FIG. 6). On the basis of the cell culture work evaluating thymidine incorporation and Western blotting (FIGS. 4 and 6), clone 967 was chosen for further evaluation of RNAi-based gene therapy to knockdown human EGFR gene expression.

Clone 967 produces an shRNA directed against nucleotides 2529-2557 (FIG. 4), and this target sequence is within the 700 nucleotide region of the human EGFR mRNA that is targeted by antisense RNA expressed by clone $882 .{ }^{30}$ Clone 967 and clone 882 equally inhibit thymidine incorporation in human U87 cells (FIG. 4), and this is evidence for the increased potency of RNAibased forms of antisense gene therapy. The clone 882 plasmid contains the EBNA-1/oriP gene element, ${ }^{30}$ which enables a 10-fold increase in expression of the trans-gene in cultured U87 cells. ${ }^{32}$ Therefore, the increased potency of the RNAi approach to antisense gene therapy enabled the elimination of the potentially tumorigenic EBNA-1 element in the expression plasmid.

In preparation for the animal work in an experimental brain tumor model, PILs were prepared using either shRNA clone 967 or antisense-RNA clone 882 and the HIR mAb (FIG. 1) to target human U87 cells in culture. A dose-response study was performed, comparing in parallel the knockdown effect of clones 967 and 882 (FIG. 5C). Either plasmid DNA was equally active in suppressing thymidine incorporation with an $\mathrm{ED}_{50}$ of $\sim 100 \mathrm{ng} /$ dish (FIG. 5C).

Calcium signaling in human U87 cells was used for further confirmation of the silencing of EGFR by clone 967 with HIR mAb-targeted PILs. EGF is known to evoke intracellular calcium signaling in brain tumor cells, ${ }^{54}$ and a similar response in human U87 glioma cells was previously reported. ${ }^{41}$ Changes in $\left[\mathrm{Ca}^{2+}\right]_{\mathrm{i}}$ were measured in response to EGF using fluorescence video microscopy. The majority of U87 cells (i.e., $>90 \%$ ) re- 

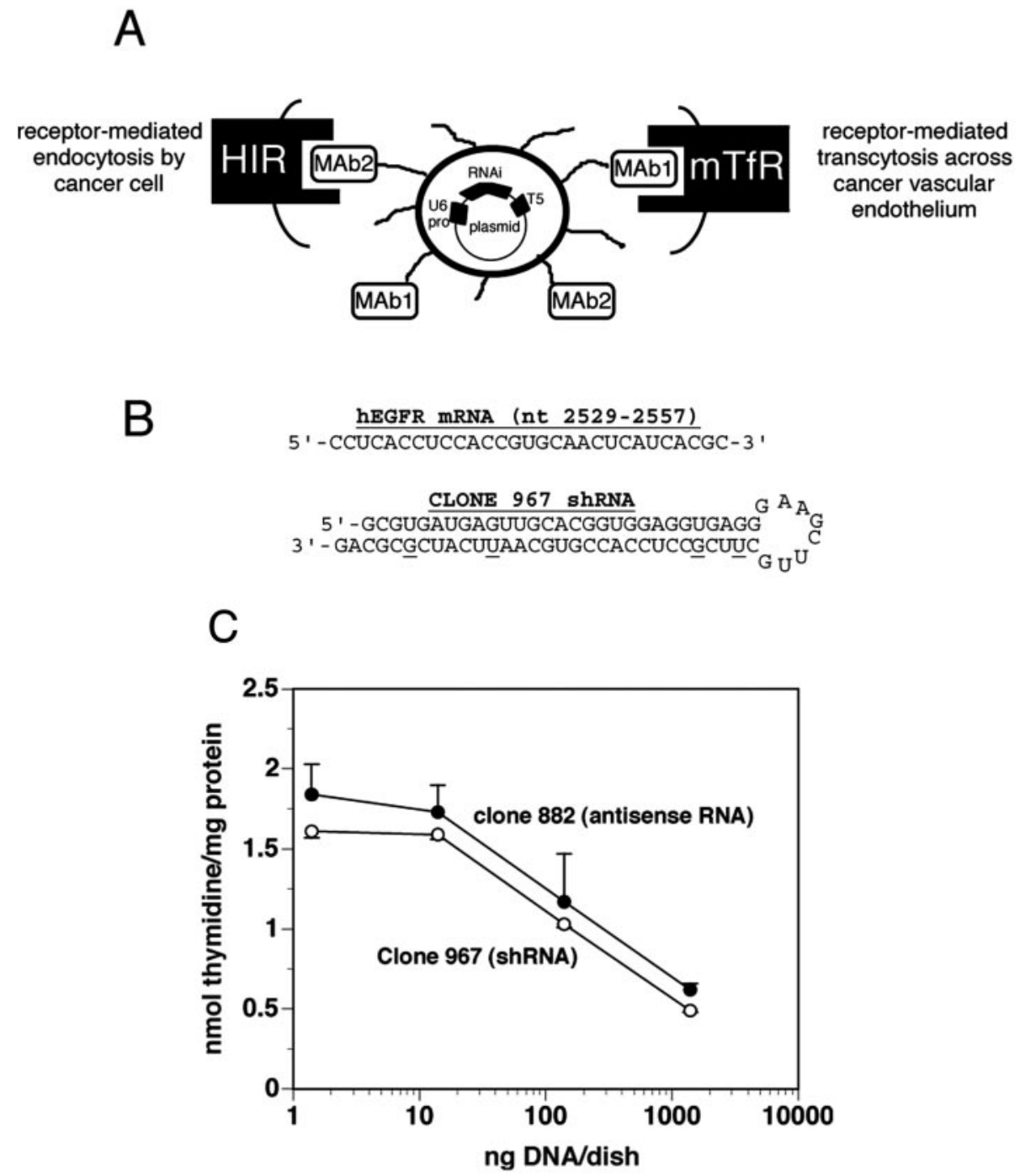

FIG. 5. Delivery of EGFR RNAi genes with pegylated immunoliposomes. A: Model of PIL that is doubly targeted to both the mouse transferrin receptor (mTfR) with the 8D3 monoclonal antibody (mAb1) and to the human insulin receptor (HIR) with the 8314 monoclonal antibody (mAb2). Encapsulated in the interior of the PIL is the plasmid DNA encoding the shRNA, which produces the RNAi. The gene encoding the shRNA is driven by the U6 promoter (pro) and is followed on the $3^{\prime}$-end with the T5 termination sequence for the U6 RNA polymerase. B: Nucleotide sequence of the human EGFR (hEGFR) sequence between nucleotides 2529 and 2557 is shown on top. The sequence and secondary structure of the shRNA produced by clone 967 is shown on the bottom. The antisense strand is $5^{\prime}$ to the eight-nucleotide loop, and the sense strand is $3^{\prime}$ to the loop. The sense strand contains $4 \mathrm{G} / \mathrm{U}$ mismatches to reduce the Tm of hybridization of the stem loop structure; the sequence of the antisense strand is $100 \%$ complementary to the target mRNA sequence. C: Human U87 glioma cells were incubated with $\left[{ }^{3} \mathrm{H}\right]$-thymidine for a $48 \mathrm{~h}$ period that follows a 5-day period of incubation of the cells with HIR mAb-targeted PILs carrying either clone 967 or 882 plasmid DNA. A dose of 1.4, 14, 140, or 1400 ng plasmid DNA per dish was used in each experiment. Data are mean \pm SEM $\left(n=3\right.$ dishes). Reproduced with permission ${ }^{41}$ (see Table 1 legend).

spond to EGF $(200 \mathrm{ng} / \mathrm{ml})$ with an increase in $\left[\mathrm{Ca}^{2+}\right]_{\mathrm{i}}$ that begins $10-30 \mathrm{~s}$ after exposure to EGF and continues for 60-300 s (FIG. 7). Treatment of U87 cells with 0.125 $\mu \mathrm{g} /$ dish clone 967 DNA in HIRmAb-PILs resulted in a significant reduction in the number of cells responding to EGF, whereas treatment with 0.25-1.5 $\mu \mathrm{g}$ DNA of clone 967 abolished the $\mathrm{Ca}^{2+}$ response to EGF in nearly all cells (FIG. 7). Clone 967 knocked down EGFR function in a dose-dependent mechanism, with respect to inhibition of both calcium flux (FIG. 7) and thymidine incorporation (FIG. 4) with an $\mathrm{ED}_{50}$ of $\sim 100 \mathrm{ng}$ plasmid DNA/dish.
For the in vivo brain cancer model, human U87 glioma cells were implanted in the caudate-putamen nucleus of adult immunodeficient scid mice. ${ }^{41}$ Without treatment, this model causes death at 14-20 days secondary to the growth of large intracranial tumors. Starting on day 5 post-implantation, mice were treated with weekly intravenous injections of either saline or $5 \mu \mathrm{g} /$ mouse of clone 967 plasmid DNA encapsulated in PILs. This PILs were doubly targeted with the 8314 murine mAb to the HIR and the $8 \mathrm{D} 3$ rat $\mathrm{mAb}$ to the mouse TfR (FIG. $5 \mathrm{~A}$ ). The saline-treated mice died between 14 and 20 days post implantation with an $\mathrm{ED}_{50}$ of 17 days (FIG. 8). The mice 


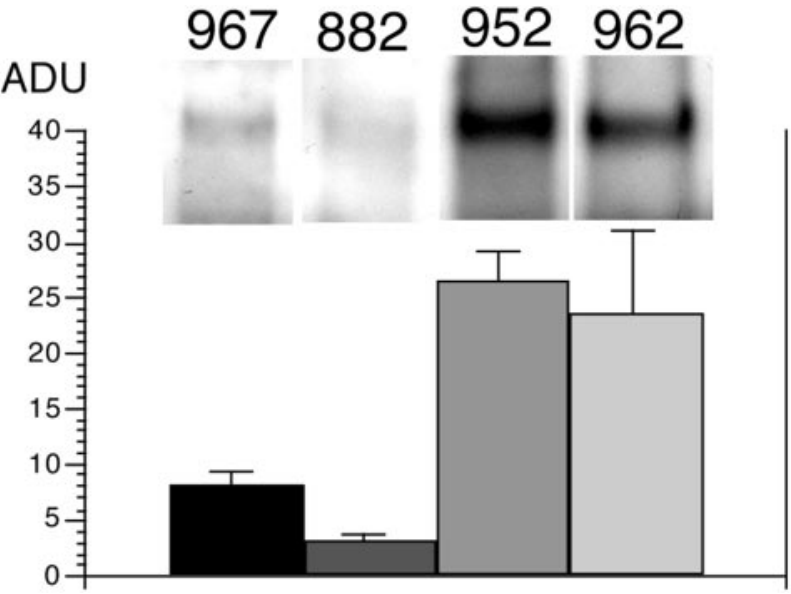

FIG. 6.. EGFR Western blotting. U87 human glioma cells were exposed to clone 967, clone 882, clone 952, or clone 962 plasmid DNA for $48 \mathrm{~h}$ and harvested for EGFR Western blotting. Arbitrary densitometric units (ADU) were computed for each treatment group. A representative scan is shown at the top of each mean \pm SEM. ( $n=3-4$ dishes). Reproduced with permis$\operatorname{sion}^{41}$ (see Table 1 legend).

treated with intravenous gene therapy died between 31 and 34 days post-implantation with an $\mathrm{ED}_{50}$ of 32 days, which represents an $88 \%$ increase over the $\mathrm{ED}_{50}$ in the saline treated animals (FIG. 8).

The silencing of the EGFR by clone 967 encapsulated in the HIR mAb- and TfR mAb-PILs was also demonstrated in vivo, as confocal microscopy showed a downregulation of the immunoreactive EGFR. ${ }^{41}$ Other evi-

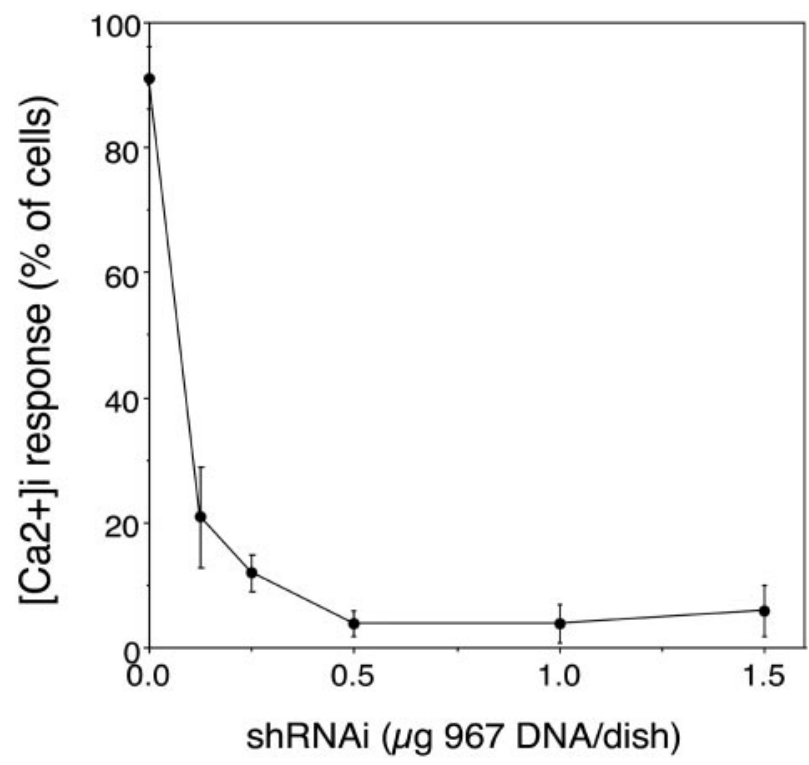

FIG. 7. Knockdown of EGFR-mediated calcium signaling by RNAi. Maximum Fluo-4 fluorescence in cultured U87 human glioma cells is shown after stimulation with $200 \mathrm{ng} / \mathrm{ml}$ human EGF. Before measurement of calcium-induced fluorescence the cells were preincubated for $24 \mathrm{~h}$ with either vehicle or HIR mAbtargeted PILs carrying clone 967 plasmid DNA. The $[\mathrm{Ca} 2+]_{\mathrm{i}}$ response in quantified by the number of cells responding to the EGF stimulus. Reproduced with permission ${ }^{41}$ (see Table 1 legend).

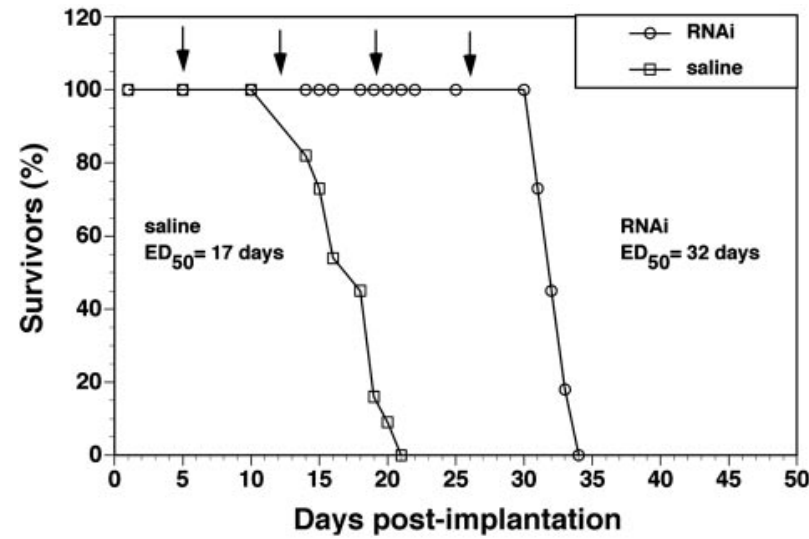

FIG. 8. Survival study. Intravenous RNAi gene therapy directed at the human EGFR is initiated at 5 days after implantation of 500,000 U87 cells in the caudate putamen nucleus of scid mice, and weekly intravenous gene therapy is repeated at days 12, 19, and 26 (arrows). The control group was treated with saline on the same days. There are 11 mice in each of the two treatment groups. The time at which $50 \%$ of the mice were dead $\left(E_{50}\right)$ is 17 days and 32 days in the saline and RNAi groups, respectively. The RNAi gene therapy produces an $88 \%$ increase in survival time, which is significant at the $p<0.005$ level (Fisher's exact test). Reproduced with permission ${ }^{41}$ (see Table 1 legend).

dence for the suppression of the EGFR in the tumor in vivo was a $72-80 \%$ reduction in tumor vascular density in the tumors of mice treated with anti-EGFR gene therapy as compared with the vascular density of brain tumors in mice treated with saline (Table 1). The EGFR has a proangiogenic function in cancer, ${ }^{55}$ and suppression of EGFR function in brain tumors results in a reduction in vascularization of the tumor (Table 1). The reduction in tumor vascular density is not a nonspecific effect of PIL administration because there is no reduction in vascular density in control mouse brain (Table 1). A basic local alignment and search tool analysis of nucleotide sequences of the human EGFR mRNA (accession number X00588) and the mouse EGFR mRNA (accession number AF275367) showed that there is only $76 \%$ identity in the mouse sequence corresponding to $2529-2557$ of the

TABLE 1. Capillary Density in Brain Tumor and Normal Brain

\begin{tabular}{lcc}
\hline Region & Treatment & $\begin{array}{r}\text { Capillary Density per } \\
0.1 \mathrm{~mm}^{2}\end{array}$ \\
\hline Tumor center & Saline & $15 \pm 2$ \\
Tumor periphery & RNAi & $3 \pm 0$ \\
& Saline & $29 \pm 4$ \\
Normal brain & RNAi & $8 \pm 1$ \\
& Saline & $35 \pm 1$ \\
& RNAi & $33 \pm 1$
\end{tabular}

Mean \pm SE $(n=15$ fields analyzed from three mice in each of the treatment groups). Reproduced with permission from Zhang et al. Intravenous RNA interference gene therapy targeting the human epidermal growth factor receptor prolongs survival in intracranial brain cancer. Clin Cancer Res 10:3667-3677. Copyright ${ }^{\odot} 2004$, American Association for Cancer Research. ${ }^{41}$ All rights reserved. 
human EGFR. Therefore, the shRNA produced by clone 967 would not be expected to effect endogenous mouse EGFR expression.

The RNAi gene therapy of brain tumors shows an $88 \%$ increase in survival time with weekly intravenous gene therapy using clone 967 encapsulated in HIR mAb- and TfR mAb-PILs (FIG. 8). This increase in survival time is not a nonspecific effect of PIL administration because prior work has shown no change in survival with the weekly administration of PILs carrying a luciferase expression plasmid. ${ }^{44}$ The increase in survival obtained with weekly intravenous anti-EGFR gene therapy is comparable to the prolongation of survival time in mice treated with high daily doses of the EGFR-tyrosine kinase inhibitor Iressa. ${ }^{22}$ However, Iressa is only active in humans carrying particular somatic mutations of the EGFR, ${ }^{20,21}$ and it was not effective in the treatment of brain cancer expressing mutant forms of the EGFR (i.e., EGFR vIII). ${ }^{22}$ Many primary and metastatic brain cancers express mutations of the human EGFR, ${ }^{56,57}$ and it is possible to design RNAi-based gene therapy that will knock down both wild-type and mutant EGFR mRNAs.

\section{FUTURE DIRECTIONS}

Recent developments in the gene therapy field demonstrate that it is possible to engineer RNAi delivery systems to silence the expression of human EGFR in intracranial tumors. ${ }^{41,44}$ This novel formulation, comprised of shRNA expression vectors and PILs, presents advantages over conventional therapeutics targeting the EGFR both in terms of specificity and transport to brain tumors via the vascular route. The shRNA expression gene therapy is also preferred over previous antisense expression plasmids ${ }^{29,30}$ because the RNAi formulation lacks the oriP/EBNA-1 elements that may represent a concern for the application of these therapeutics to humans.

The ectopic expression of shRNA genes in noncancer cells may not be desired, and it may be eliminated using tissue-specific promoters, which has been already reduced to practice in preclinical studies in either mice $e^{42}$ or primates. ${ }^{45}$ It may also be possible to restrict therapeutic gene expression to the cancer cell by placing the gene under the influence of a promoter taken from a gene selectively expressed in brain cancer. Alternatively, many solid cancers express mutant forms of the EGFR, which are produced from aberrantly processed mRNAs that contain nucleotide sequences not found in normal cells. ${ }^{58}$ These sequences may be used as shRNA targets to selectively knock down mutant transcripts in cancer cells. The shRNA expression vectors may also be designed to target a single nucleotide polymorphism. ${ }^{59}$

The weekly intravenous RNAi gene therapy directed against the human EGFR causes an $88 \%$ increase in survival time in adult mice with intracranial human brain cancer $^{41}$ (FIG. 8). The high therapeutic efficacy of the PIL-RNAi gene transfer technology is possible because this approach delivers therapeutic genes to brain via the transvascular route through the BBB. The effectiveness of this technology could be enhanced as new target genes are discovered, as well as by the simultaneous use of RNAi to knock down tumorigenic genes and gene replacement of mutated tumor suppressor genes in brain cancer. This technology has been successfully used for gene replacement of tyrosine hydroxylase in a rat model of Parkinson's disease. ${ }^{46,50}$ The efficacy of the PIL nonviral gene transfer technology has also been demonstrated in primates with levels of gene expression in brain that are 50-fold greater than those levels of gene expression in rodent brain, due to increased nuclear targeting effectiveness of the HIR mAb-PIL construct. ${ }^{45}$ Future clinical applications of the PIL approach to gene therapy of brain cancer should enable targeting of the therapeutic gene to the cancer cell with 1) minimal general toxicity and 2) absence of immunogenic response. The former has been demonstrated in experimental animals wherein the weekly administration of PILs has no toxic effects and causes no inflammation of the brain. ${ }^{60}$ PILs can be engineered with genetically modified monoclonal antibodies to deliver therapeutic genes to human brain cancer. A human-mouse chimeric HIR mAb has the same activity in terms of binding to the human BBB in vitro, or transport across the primate $\mathrm{BBB}$ in vivo, as the original murine $\mathrm{mAb} .{ }^{61}$ Even though PIL-EGFRRNAi complexes may be constructed with the chimeric HIR mAb, as the FDA has approved chimeric mAbs for human use [i.e., Inflixmab or Remicade (Centocor, Malvern, PA) for rheumatoid arthritis], it is possible to engineer these PILs with fully humanized mAbs that can be used in prolonged therapeutic treatments. The availability of humanized mAb directed to human BBB and brain cell-specific receptors may accelerate the application of this technology to the treatment of brain tumors in humans.

Acknowledgments: This work was supported in part by National Institutes of Health Grant R43-CA-109782.

\section{REFERENCES}

1. Gupta N. Current status of viral gene therapy for brain tumours. Expert Opin Investig Drugs 9:713-726, 2000.

2. Mahaley MS Jr, Mettlin C, Natarajan N, Laws ER Jr, Peace BB. National survey on patterns of care for brain-tumor patients. Neurosurgery $71: 826-836,1989$.

3. Kim TS, Halliday AL, Hedley-Whyte ET, Convery K. Correlates of survival and the Daumas-Duport grading system for astrocytomas. J Neurosurg 74:27-37, 1991.

4. Croteau D, Mikkelsen T. Adults with newly diagnosed high-grade gliomas. Curr Treat Options Oncol 6:507-515, 2001.

5. Fiveash JB, Spencer SA. Role of radiation therapy and radiosurgery in glioblastoma multiforme. Cancer J 3:222-229, 2003. 
6. McWilliams RR, Brown PD, Buckner JC, Link MJ, Markovic SN. Treatment of brain metastases from melanoma. Mayo Clin Proc 78:1529-1536, 2003.

7. Taimur S, Edelman MJ. Treatment options for brain metastases in patients with non-small-cell lung cancer. Curr Oncol Rep 5:342346, 2003.

8. Krishnan S, Rao RD, James CD, Sarkaria JN. Combination of epidermal growth factor receptor targeted therapy with radiation therapy for malignant gliomas. Front Biosci 8:1-13, 2003.

9. Kuan CT, Wikstrand CJ, Bigner DD. EGF mutant receptor vIII as a molecular target in cancer therapy. Endocr Relat Cancer 8:8396, 2001.

10. Kleihues P, Ohgaki H. Primary and secondary glioblastomas from concept to clinical diagnosis. Neuro-oncol 1:44-51, 1999.

11. Nicholson RI, Gee JM, Harper ME. EGFR and cancer prognosis. Eur J Cancer 37[Suppl 4]:S9-S15, 2001.

12. Thomas C, Ely G, James CD, et al. Glioblastoma-related gene mutations and over-expression of functional epidermal growth factor receptors in SKMG-3 glioma cells. Acta Neuropathol (Berl) 101:605-615, 2001.

13. Huncharek M, Kupelnick B. Epidermal growth factor receptor gene amplification as a prognostic marker in glioblastoma multiforme results of a meta-analysis. Oncol Res 12:107-112, 2000.

14. Yoon KS, Lee MC, Kang SS, et al. p53 mutation and epidermal growth factor receptor overexpression in glioblastoma. $J$ Korean Med Sci 16:481-488, 2001.

15. Schober R, Bilzer T, Waha A, et al. The epidermal growth factor receptor in glioblastoma genomic amplification, protein expression, and patient survival data in a therapeutic trial. Clin Neuropathol 14:169-174, 1995.

16. Shinojima N, Tada K, Shiraishi S, et al. Prognostic value of epidermal growth factor receptor in patients with glioblastoma multiforme. Cancer Res 63:6962-6970, 2003.

17. Pardridge WM. Drug and gene delivery to the brain the vascular route. Neuron 36:555-558, 2002.

18. Zhang RD, Price JE, Fujimaki T, Bucana CD, Fidler IJ. Differential permeability of the blood-brain barrier in experimental brain metastases produced by human neoplasms implanted into nude mice. Am J Pathol 141:1115-1124, 1992.

19. Senior K. Gleevec does not cross blood-brain barrier. Lancet Oncol 4:198, 2003.

20. Paez JG, Janne PA, Lee JC, Tracy S, Greulich H, et al. EGFR mutations in lung cancer correlation with clinical response to gefitinib therapy. Science 304:1497-1500, 2004.

21. Lynch TJ, Bell DW, Sordella R, Gurubhagavatula S, Okimoto RA, et al. Activating mutations in the epidermal growth factor receptor underlying responsiveness of non-small-cell lung cancer to gefitinib. N Engl J Med 350:2129-2139, 2004.

22. Heimberger AB, Leran CA, Archer GE, McLendon RE, Chewning TA, et al. Brain tumors in mice are susceptible to blockade of epidermal growth factor receptor (EGFR) with the oral, specific, EGFR-tyrosine kinase inhibitor ZD1839 (iressa). Clin Cancer Res 8:3496-3502, 2002.

23. Sridhar SS, Seymour L, Shepherd FA. Inhibitors of epidermalgrowth-factor receptors a review of clinical research with a focus on non-small-cell lung cancer. Lancet Oncol 4:397-406, 2003.

24. Sampson JH, Crotty LE, Lee S, et al. Unarmed, tumor-specific monoclonal antibody effectively treats brain tumors. Proc Natl Acad Sci USA 97:7503-7508, 2000.

25. Bendell JC, Domchek SM, Burstein HJ, Harris L, Younger J, et al. Central nervous system metastases in women who receive trastuzumab-based therapy for metastatic breast carcinoma. Cancer 97: 2972-2977, 2003.

26. Ram Z, Culver WM, Oshiro EM, Viola JJ, DeVroom HL, et al. Therapy of malignant brain tumors by intratumoral implantation of retroviral vector-producing cells. Nat Med 3:1354-1361, 1997.

27. Schlachetzki F, Zhang Y, Boado RJ, Pardridge WM. Gene therapy of the brain The trans-vascular approach. Neurology 62:12751281, 2004.

28. Pardridge WM. Drug and gene targeting to the brain with molecular Trojan horses. Nat Rev Drug Discov 1:131-139, 2002.

29. Zhang Y, Zhu C, Pardridge WM. Antisense gene therapy of brain cancer with an artificial virus gene delivery system. Mol Ther 6:67-72, 2002.

30. Zhang Y, Lee HJ, Boado RJ, Pardridge WM. Receptor-mediated delivery of an antisense gene to human brain cancer cells. J Gene Med 4:183-194, 2002.

31. Makrides SC. Components of vectors for gene transfer and expression in mammalian cells. Protein Expr Purif 17:183-202, 1999.

32. Zhang Y, Boado RJ, Pardridge WM. Marked enhancement in gene expression by targeting the human insulin receptor. J Gene Med 5:157-163, 2003.

33. Snudden DK, Smith PR, Lai D, Ng MH, Griffin BE. Alterations in the structure of the EBV nuclear antigen, EBNA1, in epithelial cell tumours. Oncogene 10:1545-1552, 1995.

34. McManus M, Sharp P. Gene silencing in mammals by small interfering RNAs. Genetics 3:737-747, 2002.

35. Couzin J. Breakthrough of the year. Small RNAs make a big splash. Science 298:2296-2297, 2002.

36. Paddison P, Caudy A, Bernstein E, Hannon G, Conklin D. Short hairpin RNAs (shRNAs) induce sequence-specific silencing in mammalian cells. Genes Dev 16:948-958, 2002.

37. Sui G, Soohoo C, Affar E, Gay F, Shi, Y, et al. A DNA vectorbased RNAi technology to suppress gene expression in mammalian cells. Proc Natl Acad Sci USA 99:5515-5520, 2002.

38. Elbashir SM, Harborth J, Weber K, Tuschl T. Analysis of gene function in somatic mammalian cells using small interfering RNAs. Methods 26:199-213, 2002.

39. Brummelkamp TR, Bernards R, Agami R. A system for stable expression of short interfering RNAs in mammalian cells. Science 296:550-553, 2002.

40. Abbas-Terki T, Blanco-Bose W, Deglon N, Pralong W, Aebischer P. Lentiviral-mediated RNA interference. Hum Gene Ther 13: 2197-2201, 2002.

41. Zhang Y, Zhang YF, Bryant J, Charles A, Boado RJ, Pardridge WM. Intravenous RNA interference gene therapy targeting the human epidermal growth factor receptor prolongs survival in intracranial brain cancer. Clin Cancer Res 10:3667-3677, 2004.

42. Shi N, Zhang Y, Boado RJ, Zhu C, Pardridge WM Brain-specific expression of an exogenous gene after i.v. administration. Proc Natl Acad Sci USA 98:12754-12759, 2001.

43. Shi N, Boado RJ, Pardridge WM. Receptor-mediated gene targeting to tissues in the rat in vivo. Pharm Res 18:1091-1095, 2001.

44. Zhang Y, Boado RJ, Pardridge WM. In vivo knockdown of gene expression in brain cancer with intravenous RNAi in adult rats. J Gene Med 5:1039-1045, 2003.

45. Zhang Y, Schlachetski F, Pardridge WM. Global non-viral gene transfer to the primate brain following intravenous administration. Mol Ther 7:11-17, 2003.

46. Zhang Y, Schlachetzki F, Zhang YF, Boado RJ, Pardridge WM. Normalization of striatal tyrosine hydroxylase and reversal of motor impairment in experimental parkinsonism with intravenous nonviral gene therapy and brain-specific promoter. Hum Gene Ther 15:339-350, 2004.

47. Boado RJ, Pardridge WM. Ten nucleotide cis element in the $3^{\prime}$ untranslated region of the GLUT1 glucose transporter mRNA increases gene expression via mRNA stabilization. Mol Brain Res 59:109-113, 1998

48. Yao D, Jiang D, Huang Z, Lu J, Tao Q, et al. Abnormal expression of hepatoma and alteration of $\gamma$-glutamyl transferase gene methylation status in patients with hepatocellular carcinoma. Cancer 88:761-769, 2000.

49. Morgenstern K, Hanson-Painton O, Wang B, De Bault L. Densitydependent regulation of cell surface $\gamma$-glutamyl transpeptidase in cultured glial cells. J Cell Physiol 150:104-115, 1992.

50. Zhang Y, Calon F, Zhu C, Boado RJ, Pardridge WM. Intravenous nonviral gene therapy causes normalization of striatal tyrosine hydroxylase and reversal of motor impairment in experimental parkinsonism. Hum Gene Ther 14:1-12, 2003.

51. McManus MT, Petersen CP, Haines BB, Chen J, Sharp PA. Gene silencing using micro-RNA designed hairpins. RNA 8:842-850, 2002.

52. Yu JY, Taylor J, DeRuiter SL, Vojtek AB, Turner DL. Simultaneous inhibition of GSK $3 \alpha$ and GSK3 $\beta$ using hairpin siRNA expression vectors. Mol Ther 7:228-236, 2003. 
53. Ewald JA, Coker KJ, Price JO, Staros JV, Guyer CA. Stimulation of mitogenic pathways through kinase-impaired mutants of the epidermal growth factor receptor. Exp Cell Res 268:262-273, 2001.

54. Hernandez M, Barrero MJ, Crespo MS, Nieto ML. Lysophosphatidic acid inhibits $\mathrm{Ca}^{2+}$ signaling in response to epidermal growth factor receptor stimulation in human astrocytoma cells by a mechanism involving phospholipase $\mathrm{C} \gamma$ and a $\mathrm{G} \alpha_{\mathrm{i}}$ protein. J Neurochem $75: 1575-1582,2000$.

55. Abe T, Terada K, Wakimoto H, Inoue R, Tyminski E, et al. PTEN decreases in vivo vascularization of experimental gliomas in spite of proangiogenic stimuli. Cancer Res 63:2300-2305, 2003.

56. Luwor RB, Johns TG, Murone C, Huang HJ, Cavenee WK, et al. Monoclonal antibody 806 inhibits the growth of tumor xenografts expressing either the de2-7 or amplified epidermal growth factor receptor (EGFR) but not wild-type EGFR. Cancer Res 61:53555361, 2001.
57. Lal A, Glazer CA, Martinson HM, Friedman HS, Archer GE, et al. Mutant epidermal growth factor receptor up-regulates molecular effectors of tumor invasion. Cancer Res 62:3335-3339, 2002.

58. Luo X, Gong X, Tang CK. Suppression of EGFRvIII-mediated proliferation and tumorigenesis of breast cancer cells by ribozyme. Int J Cancer 104:716-721, 2003.

59. Miller VM, Gouvion CM, Davidson BL, Paulson HL. Targeting Alzheimer's disease genes with RNA interference an efficient strategy for silencing mutant alleles. Nucleic Acids Res 32:661668, 2004.

60. Zhang Y-F, Boado RJ, Pardridge WM. Absence of toxicity of chronic weekly intravenous gene therapy with pegylated immunoliposomes. Pharm Res 20:1779-1785, 2003.

61. Coloma MJ, Lee HJ, Kurihara A, Landaw EM, Boado RJ, et al. Transport across the primate blood-brain barrier of a genetically engineered chimeric monoclonal antibody to the human insulin receptor. Pharm Res 17:266-274, 2000. 